\section{Pathophysiologie des Asthma bronchiale} Leukotriene im Zentrum

\section{Leukotriene sind maßgeblich an der Pathophysiologie des Asthma bronchiale beteiligt. Sie bewirken eine Bronchokonstriktion und sind an der Entzündungsreaktion beteiligt. Asthmatiker haben generell erhöhte Leukotrienwerte im Plasma und weitere Konzen- trationsanstiege sind z.B. nach Allergen- oder Acetylsalicylsäure- provokation oder während einer Exazerbationsphase nachweisbar.}

\begin{abstract}
E $s$ ist noch gar nicht so lange her - Anfang der 70er Jahre, dass eine Gruppe hochaktiver Fettsäurederivate isoliert, chemisch definiert und mit dem Namen Leukotriene belegt wurden. Diese Leukotriene sind an einer ganzen Reihe von physiologischen Vorgängen im Organismus beteiligt - bei Lungenkrankheiten kommt ihnen vor allem als Effektormediatoren mit bronchokonstriktorischer Wirkung ein hoher pathogenetischer Stellenwert zu.

Mit der Entwicklung spezifischer, die Leukotrienwirkung hemmender Substanzen erhielt man detaillierte Kenntnisse nicht nur über die bronchokonstriktorischen Wirkungen von Leukotrienen, sondern auch über ihren Anteil an der inflammatorischen Komponente des Asthma bronchiale.
\end{abstract}

\section{Pathologische Zysteinylgruppe}

Unter Leukotrienen versteht man heute eine Gruppe von fünf biologisch aktiven Stoffwechselprodukten der Arachidonsäure, die im Rahmen entzündlicher Erkrankungen von verschiedenen Zellen durch das Enzym 5-Lipoxygenase gebildet werden. Neben dem chemisch instabilen Zwischenprodukt LTA4 gehören hierzu die eine Zysteinylgruppe tragenden Zysteinyl-Leukotriene LTC4, LTD4 und LTE4 sowie das zysteinylfreie Leukotrien LTB4.

Die Zysteinyl-Leukotriene binden am Zielorgan an zwei Zysteinyl-Rezeptoren, heute als CysLT1- und CysLT2Rezeptor bezeichnet. Zellen der glatten Atemwegsmuskulatur bilden CysLT1Rezeptoren, während die Gefäßmuskelzellen des Pulmonalkreislaufs CysLT2-Rezeptoren tragen. Die heute triene sind ausschließlich Antagonisten des CysLT1-Rezeptors.

Gerade die Cysteinyl-Leukotriene spielen in der Pathophysiologie asthmatischer Erkrankungen eine wichtige Rolle (s. Kasten). Asthmatiker zeigen z.B. einen höheren LTE4-Plasmaspiegel als nicht asthmatische Personen. Erhöhte LTE4-Werte werden auch nach Allergenprovokation- bzw. Provokation mit Acetylsalicylsäure gefunden. In zwei anderen Studien korrelierte die LTE4Ausscheidung im Urin mit dem Ausmaß der Bronchokonstriktion und den klinischen Symptomen.

Leukotrienrezeptor-Antagonisten hemmen die asthmatische Atemwegsobstruktion. Auch antientzündliche Eigenschaften werden den Leukotrienrezeptor-Antagonisten zugeschrieben, vor allem wird die Infiltration von Eosino- therapeutisch verfügbaren Antileuko-
Entzündung und Obstruktion durch Leukotriene

Die rekurrierende Atemwegsobstruktion beim Asthma bronchiale geht im wesentlichen auf folgende Pathomechanismen zurück, die u.a. durch eine erhöhte Leukotriensynthese hervorgerufen werden:

- Konstriktion der glatten Bronchialmuskulatur

- Steigerung der Kapillarpermeabilität mit Bildung von Schleimhautödemen

- vermehrte Produktion von viskösem Mukus

- Hemmung der mukoziliären Clearance

- Infiltration und Aktivierung von Entzündungszellen

Auf alle diese Mechanismen haben Leukotrien-Rezeptorantagonisten einen positiven Einfluss und sind daher für die Therapie des Asthma bronchiale prinzipiell geeignet.

philen in die Atemwege gestoppt. Ihr klinischer Nutzen in der Asthmatherapie ist durch Studien belegt, ihr Stellenwert wird in weiteren Untersuchungen derzeit erkundet.

\section{Kroegel C et al.}

Leukotriene und asthmatische Entzündung. Allergologie 22; 1999: 161-170.

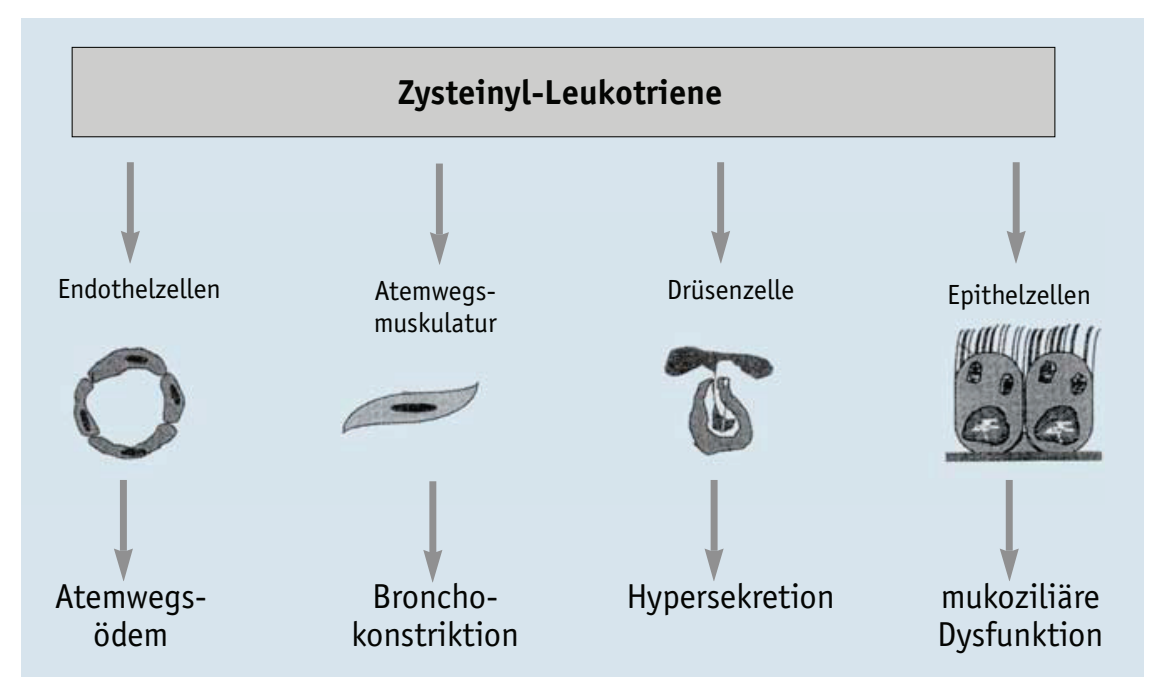

Bisher bekannte und für die Pathogenese des Asthma bronchiale relevante Wirkungen der Zysteinyl-Leukotriene (nach C. Kroegel et al. 1999). 